\title{
CBCT Analysis in osteoradionecrosis case followed by hyperbaric oxygen treatment (a case study)
}

\author{
Lusi Epsilawati*
}

\section{Abstract}

Objective: Osteoradionecrosis is an infectious disease and inflammation that occurs in the jaw after doing radio therapy. Radiographic picture of the disease is extensive visible radio lucent lesions in the jaw. In Clinical condition, it can be seen as fistula and abscess which exist in the jaw. One therapy for the treatment of this condition is the "Hyperbaric 0xygen therapy". This paper proposed to present a case report of osteoradionecrosis patient treated with hyperbaric oxygen therapy.

Methods: The patient was a 55-year-old woman who came with complaints of pain in left and right lower jaw that existed since a month ago after doing radiotherapy for 6 months. The patient was diagnosed with osteoradionecrosis. For healing, selected medical therapy was employed and the patient was advised to take hyperbaric oxygen therapy in Al Minto Harjo hospital Jakarta.

Results: One month after doing hyperbaric oxygen therapies, change and healing have been noted. Lesions formed were still broad but it disappeared, abscess dried, necrotic tissue disappeared or reduced as well as the formation of new bone matrix.

Conclusion: Obviously able to heal lesions caused osteoradionecrosis and osteomyelitis by hyperbaric oxygen therapy.
Department of Dentomaxillofacial Radiology, Faculty of Dentistry, Padjadjaran University, Bandung, Indonesia

Corresponding Author: Lusi Epsilawati, Department of Dentomaxillofacial Radiology, Faculty of Dentistry, Padjadjaran University, Bandung, Indonesia lusifkg92@gmail.com

Received: 16 March 2016

Revised: 25 April 2016

Accepted: 26 April 2016

Available Online: 30 April 2016

Keywords: Cone beam computed tomography, Hyperbaric oxygen therapy

Cite this Article: Epsilawati L. 2016. (BCT Analysis in osteoradionecrosis case followed by hyperbaric oxygen treatment (A case study). Journal of Dentomaxillofacial Science 1(1): 67-72. D01:10.15562/jdmfs.v1i1.28

\section{Introduction}

Osteoradionecrosis (ORN) is the term of tissue categorized by the occurrence of necrotic tissue after performing radiotherapy. It is the complication mostly frightened due to application of radiotherapy in the neck and head cancer cases. ${ }^{1-3}$ Actually, ORN can occur only when the radiation therapy is performed with dose of radiation ranges 50-60 gray, whereas the tissues usually will turn out to be dead spontaneously in above 60 gray. The prevalence which reveals about the ORN occurrence from 1970 until now only revolves to about 5.4-11.8\%.

In the histological aspect, ORN consists of lenient tissue and necrotic bone. ${ }^{1,2}$ The occurrence starts after radiation therapy. The tissues undergo hypoxia caused by damaging the capillary blood vessels in normal tissues and then generally ruin the vascularization in the tissues. This term causes death in the collagen cells and culminates into cell death. The body actually responds to this term by forming the new cells, however, the lack of oxygen occurs because of the diminished vascularization in the areas so that the process of cell necrosis is faster than the formation of new cells. ORN clinically will appear as an unrecovered wound. ORN does not occur in every individual, it depends on the individual's reaction against radiation. ORN more often occurs on mandible because it naturally has minimal vascularization and higher dose than maxilla. ${ }^{1,4,5}$
The imaging process is highly necessary for the analysis in ORN case therefore some instruments are required to describe it well. The instruments that are greatly administered for this purpose are panoramic, $\mathrm{CBCT}$ and $\mathrm{CT}$ whereas the more appropriate modality is CT but unfortunately to install this equipment requires a lot of expenses. CBCT is the instrument which is the most likely used to analyze the well-detailed depiction because of its three dimensions advantage. ${ }^{6-10}$

\section{Case Report}

A 55-year-old woman came to Dental Hospital in Bandung, Indonesia complained about the swelling and pain in all part of her mandible. This complaint had occurred for six months after performing the radiotherapy for nasopharyngeal tumor case. Generally the patient was in a limp and painful condition. The extra-oral examination showed the extensive swelling in the mandible caused a change of her face shape, lenient consistency and when pressed, the patient felt pain, warm and reddish. The intra-oral examination showed the swelling on all mandible regions both left and right anterior and posterior. To complete the diagnosis, panoramic examination figure 1 and radiograph CBCT were conducted and the result showed that there were lesions: A. Locations left 
and right mandible from the first premolar region until the third molar, B. Margin unlimited/irregular, C. Internal structure: radiolucent, multiple, D. The effect against around tissue: the bone around the lesion area appeared vanished/resorption.

Table 1 at the time, the patient was treated with antibiotics, analgesic and roburatia. The patient was advised to undergo the hyperbaric treatment in
AL Mintohardjo Hospital, Jakarta. Three months after the treatment, the patient returned to conduct CBCT and evidently, there was significant improvement. The interpretation revealed.

\section{Discussion}

Osteoradionecrosis or ORN is bone necrosis affected by radiotherapy. ${ }^{12}$ The abnormality can be

Table 1 The examination results of patient before and after hyperbaic oxygen treatment.

\section{Before treatment \\ The examination on left mandible}

CT imaging:

Extensive lesion in the mandible cortical bone was disconnected.

\section{Axial:}

There was lesion on axial and it was $26.8 \mathrm{~mm}$ long and $8.2 \mathrm{~mm}$ wide, which was observed as the largest.

\section{Sagital:}

There was extensive radiolucent lesion on mandible corpus with the limit of ill-defined and left mandible of cortical bone was disconnected. It had $6.9 \mathrm{~mm}$ in length and $20.3 \mathrm{~mm}$ in height of influenced corpus.

\section{The examination on right mandible}

\section{CT Imaging:}

On the bone surface, we could observe that circumstance was still intact without abnormality, the bone damage did not reach the anterior mandible: however, there was bone damage in linggir (?) of left anterior mandible. We could observe that the bone thickness from anterior to posterior looked diminished so that the mandible looked thinner. We estimated that the bone ran into resorpsi from bucal line.

\section{Axial:}

The range of thickness in front linggir was $4.2 \mathrm{~mm}$ long so far from normal circumstance $+/-8-9 \mathrm{~mm}$ long

Sagital:

Beginning: in sagittal section, we can observe that the quality of right mandible was more defective than that of left mandible. Trabekula(Trabecula?) from bone tissue emerged diminished and irregular. The average of bone density was $150-170 \mathrm{Hu}$

\section{Right mandible}

\section{CT imaging:}

There was bone damage, mainly in lingual right mandible. In the buccal surface of mandible, the damage occurred so that the bone was continuity disappearing in several areas. The bone damage from lingual looked extremely wide but the bone continuity of mandible was still integrated.

Axial:

The thickness of front linggir ranged $4.2 \mathrm{~mm}$ so far from normal circumstance $+/-8-9 \mathrm{~mm}$

\section{Sagittal:}

In sagittal section, we could observe that the quality of right mandible was more defective than that of left mandible. Trabekula from bone tissue appeared diminished and irregular. The average of bone density was $150-170 \mathrm{Hu}$

\section{After treatment}

CT imaging:

Improvement from initial radiograph which formed bone on lesion area. Although the cortical bone was not close yet, it appeared like the process of healing step.

\section{Axial:}

There was extensive lesion during the process of healing or the bone lesion was not formed yet.

Sagital:

The mandible cortical bone became thick and grew in various spots which categorized that healing process occurred.

\section{CT Imaging:}

After conducting the hyperbaric treatment, evidently, the anterior mandible did not show significant improvement, but bone compression quantity had shown to increase.

Axial:

We could observe that the thickness of left anterior linggir increased from $4.2 \mathrm{~mm}$ to $5.3 \mathrm{~mm}$ long. It proved that significant bone growth had occurred in buccolingual line.

Sagital:

The mandible was improved, with neat pla (?) trabekula and the density value increases from $150-170 \mathrm{Hu}$ to $250-300 \mathrm{Hu}$. It proved that the improvement also occurred in bone quality

\section{CT imaging:}

The bone circumstances, both before and after treatment, did not show significant improvement. In buccal lesion disconnected bone continuity still appeared in several areas.

From lingual side, there was quite wide bone damage from buccal to lingual and reached almost all of mandible corpus. There was no significant improvement yet.

Axial:

The thickness in the linggir left anterior increased from $4.2 \mathrm{~mm}$ to $5.3 \mathrm{~mm}$ long. It proved that significant bone growth had occurred in buccolingual line.

Sagittal View:

Mandible was improved, with neat pla trabekula and the density value increased from $150-170 \mathrm{Hu}$ to $250-300 \mathrm{Hu}$. It proved that the improvement also occurred in bone quality. 
illustrated as $3 \mathrm{H}$ effect principles from radiotherapy: hypocellular, hypovascular and hypoxia. This circumstance makes growth of connective tissue and other cells become hampered. ${ }^{13}$ Histologically, a bone is called "necrosis" when the osteocyte which normally exists disappears. Necrotic tissue in mouth cavity is quite susceptible against infection, pathological fracture and secondary infection, these three matters can provoke inflammation in the tissue, so that it can cause infection. Usually, ORN is started after minimum 3 months of treatment term. ${ }^{7-8}$ In this case we can observe that the patient is diagnosed of suffering from ORN after 6 months run for therapy. We can assume that it is related to the patient's condition, who is quite weak and it bring through the occurrence of secondary infection after nasopharyngeal tumor. Figure 2 besides that, from the CBCT results, we can assume that it had occurred in secondary fracture of left mandible. In this case, ORN occurred in mandible and proved that mandible is the most susceptible part of jowl. This matter occurred because of the quite supporting circumstance of bone structure. ${ }^{9,10}$

The clinical manifestations of ORN are pain, multiple fistulas in suffered necrotic bone areas, fracture pathology, and suppuration. Contributing factors against the ORN growth are: tooth trauma, tumor location, radiation dose, vascularization and fractionation, the interval of radiation process, nutrient, liquor and the use of tobacco, as well as chemotherapy. ${ }^{6-8}$ In this case, ORN probably occurred as a consequence of patient's condition which was not very well. The patient feels too hard to eat and drink after nasopharyngeal surgery, which makes the patient turn out to be weak and susceptible against infection. ${ }^{14-15}$

Clinically, there are three types of ORN such as: A. Type I: ORN caused by trauma after surgery, B. Type II: ORN caused by trauma for 1 year or more during the radiation therapy and the last, C. Type III: this type is also known as spontaneous

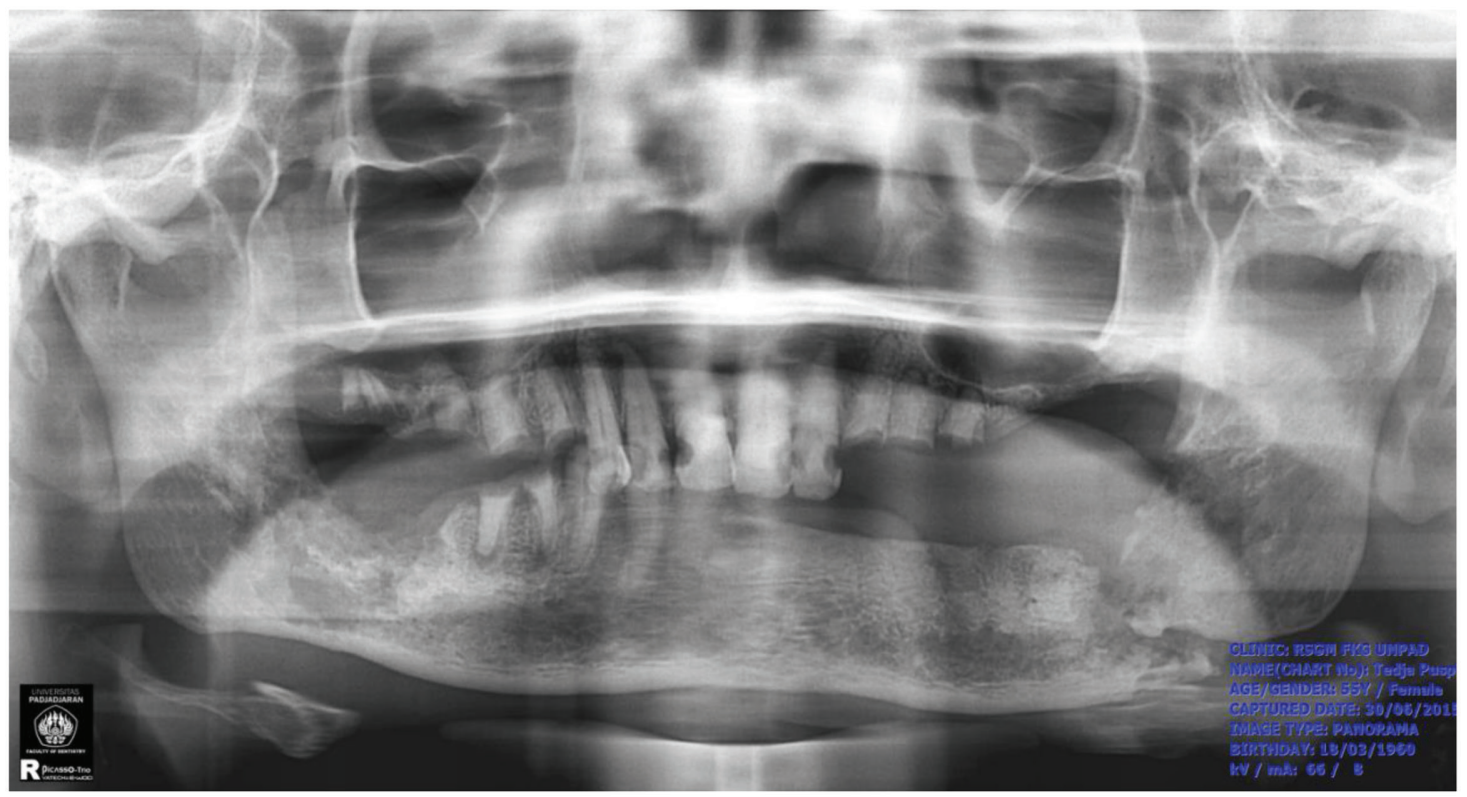

Figure 1 In the radiograph panoramic patient were found to have radiolucent lesions in mandible from the left and right regiopremolar to the mandible corpus, followed by multiple and unlimited lesion and cortical bone of left mandible which is disconnected.

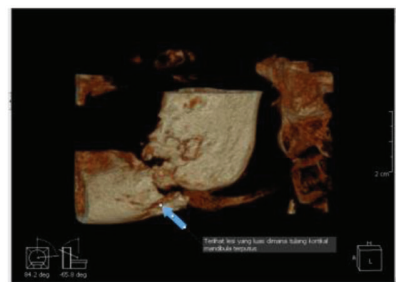

A

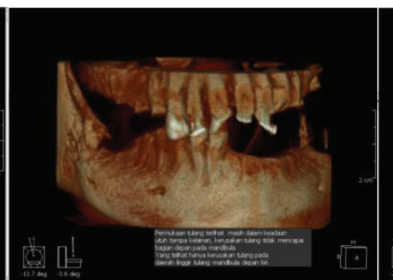

B

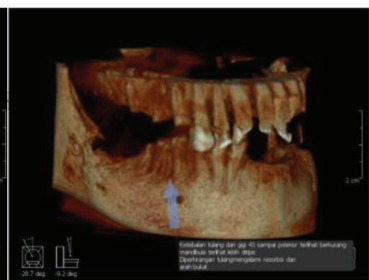

$\mathrm{C}$

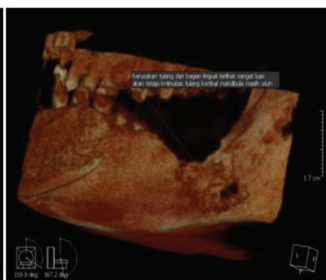

$\mathrm{D}$

Figure 2 A. The illustration of patient CT before conducting the treatment, B. Left mandible, pathologic fracture, C. Exists mandible anterior showing no lesion, but the bone becomes quite slight, $\mathrm{D}$. right mandible showing lesion and bone damage of lingual. 


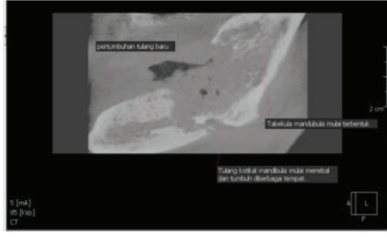

A

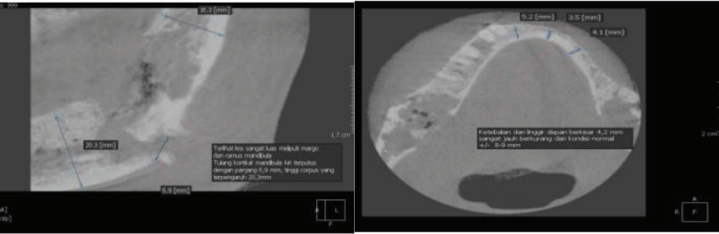

B

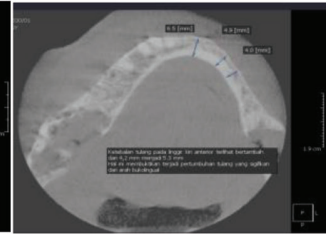

$\mathrm{D}$

Figure 3 The CBCT image of the patient (pre-and post-HBO treatment). A. In left mandible (pre-HBO) shows pathological fracture followed necrotic lesion in several parts of the lesion, B. In left mandible (post-HBO) the fracture was still existed but the necrotic tissue in the inside part of the lesion had vanished, C. The bone thickness of anterior mandible (pre-HBO) turned out to bequite slight, $\mathrm{D}$. The bone thickness of anterior mandible (post-HBO) became escalated.
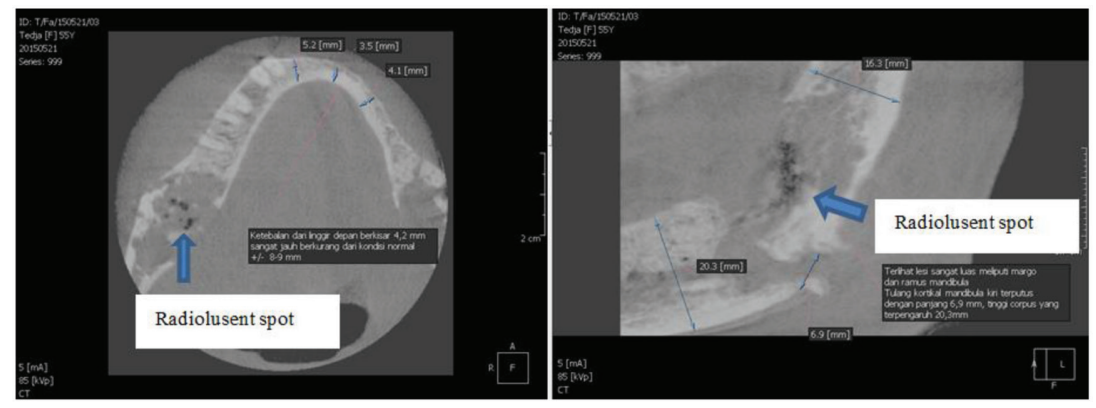

Figure 4 Radiolucent spot reveals that the tissue became partially necrotic in right and left mandible after conducting the radiotherapy, before conducting the $\mathrm{HBO}$ treatment.
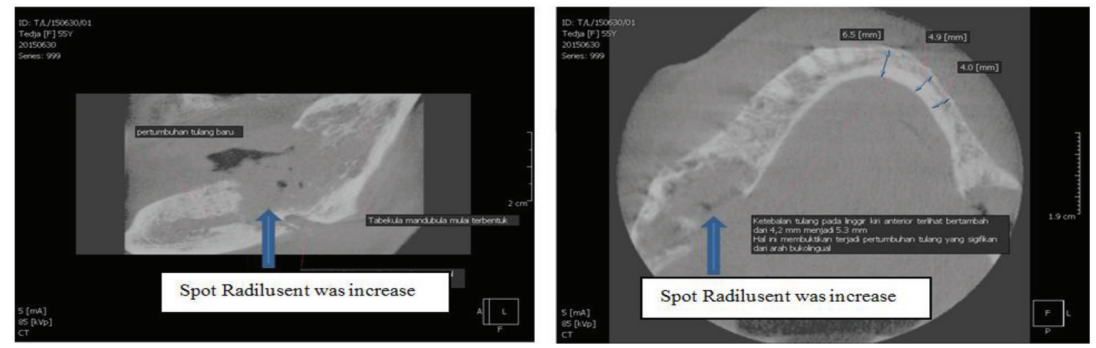

Figure 5 There is a radiolucent spot which tends to disappear after conducting the HBO treatment.

type in that ORN can occur anytime, but on the average occurs after conducting the radiotherapy for 6 months until 2 years. ${ }^{16-17}$ In this case, the secondary infection and pathological fracture occur therefore it is probably ORN type III which occurs spontaneously however, the incident is earlier than the initial research's suggestion (6 months-2 years after surgery). It can probably come about the prevalent circumstance and also due to poor nutrient of the patient.

For several years, ORN has been treated by various means, evidently the results are not ideal and successful yet. ${ }^{14}$ Hyperbaric oxygen serves as reference therapy, although it is still considered as controversial one by several specialists because the results of the therapy are still dubious. ${ }^{18-24}$ Some research clarifies that $\mathrm{HBO}$ provides fascinating result toward prophylactic before tooth extraction of the patient which is being considered as the risk of developing ORN..$^{25-28}$

Hyperbaric Oxygen Treatment (HBOT) is the treatment which administers highly pressurized oxygen more than atmospheric pressure, it is provided to escalate the recovery process of the tissue, chiefly in lesion followed fewer blood vessels. ${ }^{19-29}$ It requires the utilizations of pressurized room and sensor tool of $100 \%$ oxygen. Initially, $\mathrm{HBO}$ is administered in the treatment of decompression disease, it has quite great effectiveness in medicating gaseous gangrene and carbon-monoxide virulence. ${ }^{30} \mathrm{HBO}$ is a treatment modality which administers high oxygen pressure. The technique puts the patient on pressurized room (monoplace or chamber). The patient is put in the room for 1 hour to be provided with pure oxygen. ${ }^{1,2}$ There are three criteria where the treatment is called "successful": there is no effect in the bone, shows fistula closing (if there is), and does not show indication in other tissues. It is considered to be successful when a minimum of two criteria are completed or fulfilled. ${ }^{13}$

In this case, the prevalent circumstance of the first patient arrival was not in a good condition that she was hardly capable to eat and has soreness that she was unable to walk and came by wheelchair. There were multiple fistulas in intraoral. After running the $\mathrm{HBO}$ treatment for three months in $\mathrm{AL}$ Mintoharjo Hospital in Jakarta, the patient showed a quite significant improvement. Generally, prevalent circumstance turned out to be very well and the patient could eat and drink without obstacle and soreness and she could walk and speak very well. After the treatment, the fistel (fistulas) turned close and generally, there was no effect in her body and bone. If we refer to the stipulation, we can assume that the $\mathrm{HBO}$ treatment is successful.

Figure 3 when the tissue encounters radiation, the recovery process runs normally, where the center of lesion will be surrounded by tissues which have normal perfusion so that the process of body biochemistry turns out to be precursor for occurring 
of the initial inflammation process and finally the fibrous tissue is formed. However, this process does not run well because of the circumstance after providing radiation in that lactic acid level, iron and oxygen diminishes so that the stimulation to conduct the recovery process is difficult to form and it is added with the minimum vascularization circumstance, therefore automatically the recovery process also diminishes. ${ }^{30,31}$ In this case, the patient ran radiation and it was shown by radiograph that there was tissue death proven by the appearance of radiolucent spot in the image inside the lesion.

Figure 4 after conducting the $\mathrm{HBO}$ treatment, evidently, the radiolucent spot disappeared and changes into intermediate image of the tissue. It reveals that there is a new tissue formation process. Besides that, clinically, multifistel which existed before treatment also disappear. From the figure, we can see there was a significant change and after conducting the treatment for three months a new tissue formed as well as it proves that the HBO treatment is the suitable therapy for this case.

Figure 5 the recovery process administered by $\mathrm{HBO}$ treatment is by means of escalating the oxygen pressure which is available in all tissues and diminishes embolism and gaseous volume in the body. The effect of the escalating oxygen pressure is the increase of oxygen transportation capacity from blood. In normal atmosphere pressure, oxygen transportation is restricted by the oxygen capability to bind to hemoglobin in red blood cells. With $\mathrm{HBO}$, stimulation to escalate oxygen transportation also increases. New evidence clarifies that HBO can mobilize the parent cell/progenitor in the bone marrow through the nitric oxide mechanism so that the tissue regeneration process occurs faster. ${ }^{31}$

Hyperbaric Oxygen escalates the amount of free radical oxygen and can oxidize protein and lipid of membrane, damage DNA and obstruct the function of bacterium metabolism. HBO is quite effective against anaerobic bacteria and supports peroxide system of leukocytes in eliminating the bacteria. Lesion recovery normally occurs through hemostasis step, infection agent eradication, inflammation respond resolution, reformation of matrix connective tissue, angiogenesis and resurfacing. Chronic injury does not fully continue these processes because of least factors of local and systemic host. Failed injury in recovery process is usually hypoxia. ${ }^{17}$ Several components of recovery from injury process are also influenced by oxygen concentration. It reveals why $\mathrm{HBO}$ is the best therapy for chronic injury. ${ }^{17}$

The existing mechanism that increases the osteoclasts is by using HBO. Necrotic bone resorption by osteoclast depends on the presence of oxygen. In vivo research has proved that it becomes better in animals suffering from ORN. Besides that, as mentioned before, HBO treatment can facilitate penetration or antibiotics function. Other HBOT characteristics as clarified before are like neovascularization and obstructive inflammation respond and other possible treatment benefit. ${ }^{31}$

Cone-beam computed tomography (CBCT) is an imaging technique which produces three dimension image which has high resolution and low dose when compared to the conventional CT. CBCT exploits cone ray emission and rotating detector by capturing two dimension image and reconstructing it into three dimension image. ${ }^{31} \mathrm{CBCT}$ also provides image with small view so that the endodontists are able to administer the endodontic diagnosis requirement and monitor before and after treatment. During this time, many researchers learn to observe CBCT clinical application in dentistry therapy. ${ }^{32}$ In dentistry, CBCT turn out to be standard treatment where it is quite necessary. Taking picture in CBCT is performed by applying gantry which rotates to the source of X-ray and detector following suitable gantry movement which focuses line also known as ROI. During radiation, there are hundreds of planar images obtained in suitable field of view (FOV), minimum of $180^{\circ}$ in bow. The CBCT software will fuse all of recorded images. All FOVs require only one time rotation to reconstruct image. The CBCT is complement modality to apply particularly and does not serve as substitute for 2D modality image. The width of FOV or scanned volume, depends on the detector's size, geometry and its collimated ability. The FOV is able to cylinder rotate or circle like in Newtom 3G. ${ }^{33,34}$

\section{Conclusion}

CBCT is a quite accurate radiograph modality in evaluating the abnormality of tooth and jowl including the osteoradionecrosis by using hyperbaric oxygen therapy.

\section{References}

1. Tejashree M, Munde A, Shoeb, et al. Bilateral osteoradionecrosis of Mandible: Case report. IJIRSET 2014; 3.

2. Rathy R, Sunil S, Nivia M. Osteoradionecrosis of mandible: Case report with review of literature. Contemp Clin Dent 2013;4: 251-253.

3. Lyons A, Ghazali N. Ostoradionecrosis of the jaws: current understanding of its pathophysiology and treatment. Br J Oral Maxillofac Surg 2008;46: 653-660.

4. Murphy-Lavoie H, Butler FK, Hagan C. Hyperbaric oxygen therapy and the Eye. Undersea and Hyperbaric Medicine Society 2008.

5. Kindwall E, Whelan H. Hyperbaric Medicine Practice. 2nd ed. Flagstaff AZ: Best Publishing Company; 2004.

6. Wahl MJ. Osteoradionecrosis prevention myths. Int J Radiat Oncol Biol Phys. 2006;64: 661-669.

7. Sharma WGCDRA. Role of Hyperbaric Oxygen Therapy in Dental Surgery. Ind J Aerospace Med 2003:47; 23-29. 
8. Devaraj D, Srisakthi D. Hyperbaric Oxygen Therapy-Can be the new era in dentistry?: literature Study. J Clinical Diagnostic Research 2014;8: 263-265.

9. White SC, Pharoah. Oral Radiology: principles and interpretation, 7th ed. Canada: St. Mosby Louis Missour; 326-328.

10. Chan KC. Jaw bone changes on panoramic imaging after head and neck radiotherapy, M.Sc. thesis. University of Toronto 2012.

11. Hermans R, Fossion E, Ioannides C, et al. CT findings in osteoradionecrosis of the mandible, Skeletal Radiol 1996;25: 31-36.

12. Wong JK, Wood RE, McLean M. Conservative management of osteoradionecrosis, Oral Surg Oral Med Oral Pathol Oral Radiol Endod 1997;84: 16-21.

13. Epsilawati L. Tedja Medical Record radiointerpretation RSGM Sekeloa. Bandung; 2015.

14. Gesell, Laurie B. Hyperbaric oxygen therapy indications. The hyperbaric oxygen therapy committee report 12th ed. Durham NC: Undersea and Hyperbaric Medical Society; 2008.

15. Feldmeier J. Hyperbaric oxygen indications and results the hyperbaric oxygen therapy committee report. Kensington Maryland: Undersea and Hyperbaric Medical Society, Inc; 2003. p. $1,2,4,5,7,8$.

16. Al-Hadi H, Smerdon GR, Fox SW. Hyperbaric oxygen therapy accelerates osteoblast differentiation and promotes bone formation. J Dent 2015;43: 382-388.

17. Rambold H, Boenki J, Stritzke G, et al. Differential vestibular dysfunction in sudden unilateral hearing loss. Neurology 2005;64: 148-151.

18. Kucharzewski M, Slezak A, Franek A. Topical treatment of non-healing venous leg ulcers by cellulose membrane. Phlebologie 2003;32: 147-151.

19. Nelson EA, Bradley MD. Dressings and topical agents for arterial leg ulcers. Cochrane database of systematic Reviews 2007; 1.

20. McGuckin M, Waterman R, Brooks J, et al. Validation of venous leg ulcer guidelines in the United States and United Kingdom. Am J Surg 2002;183: 132-137.

21. Diegelmann RF, Evans MC. Wound healing: an overview of acute, fibrotic and delayed healing. Front Biosci 2004;9: 283-289.

22. Thom SR. Effects of hyperoxia on neutrophil adhesion. Undersea Hyperb Med Spring 2004;31: 123-131.

23. Chen WYJ, Rogers AA. Recent insights into the causes of chronic leg ulceration in venous diseases and implications on other types of chronic wounds. Wound Rep Reg 2007;15: 434-449.

24. D'Souza J, Goru J, Goru S, et al. The influence of hyperbaric oxygen on the outcome of patients treated for osteoradionecrosis: 8 year study. Int J Oral Maxillofac Surg 2007;36: 783-787.

25. Bui QC, Lieber M, Withers HR, et al. The efficacy of hyperbaric oxygen therapy in the treatment of radiation-induced late side effects. Int J Radiat Oncol Biol Phys 2004;60: 871-878.

26. Roeckl-Wiedmann I, Bennett M, Kranke P. Systematic review of hyperbaric oxygen in the management of chronic wounds. Br J Surg 2005;92: 24-32.

27. Escobar SJ, Slade JB Jr, Hunt TK, et al. Adjuvant hyperbaric oxygen therapy (HBO2) for treatment of necrotizing fasciitis reduces mortality and amputation rate. Undersea Hyperb Med 2005;32: 437-443.

28. Sheffield PJ, Smith APS. Physiological and pharmacological basis of hyperbaric oxygen therapy. In Bakker DJ, Cramer FS. Editors. Hyperbaric surgery. Flagstaff AZ: Best Publishing Company; 2002. p. 63-109.

29. Babul S, Rhodes EC. The role of hyperbaric oxygen therapy in sports medicine. Sports Med 2000;30: 395-403.

30. Malda J, van Blitterswijk CA, van Geffen M, et al. Low oxygen tension stimulates the redifferentiation of dedifferentiated adult human nasal chondrocytes. Osteoarthritis Cartilage 2004;12: 306-313.

31. Alshehri MA, Alamri H, Alshalhoob M. CBCT applications in dental. Cat Cam Journal 2010; 10.

32. Scarfe WC, Martin D, Levin, et al. Use of cone beam computed tomography in endodontics International Journal of Dentistry. Hindawi Publishing Corporation; 2009.

33. Farman AG. Image guidance: the present future of dental care. Practical Procedures \& Aesthetic Dentistry 2006;18: 342-344.

34. Hayakawa Y, Sano T, Sukovic P, et al. Cone beam computed tomography: a paradigm shift for clinical dentistry. Nippon Dent Review 2005;65: 125-132.

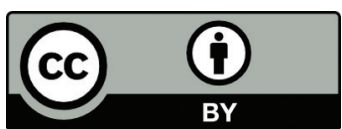

This work is licensed under a Creative Commons Attribution 